\title{
Voxel-based morphometry reveals altered gray matter volume related to cognitive dysfunctioning in neovascular glaucoma patients
}

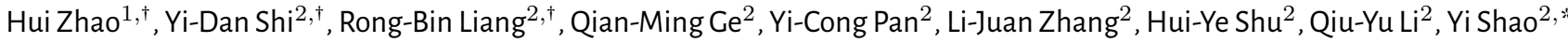 \\ ${ }^{1}$ Xinhua Hospital Affiliated to Shanghai Jiao Tong University School of Medicine, 200092 Shanghai, China \\ ${ }^{2}$ Department of Ophthalmology, The First Affiliated Hospital of Nanchang University, Jiangxi Centre of Natural Ocular Disease Clinical Research Center, \\ 330006 Nanchang, Jiangxi, China \\ *Correspondence: freebee99@163.com (Yi Shao) \\ ${ }^{\dagger}$ These authors contributed equally.
}

DOI:10.31083/j.jin2004086

This is an open access article under the CC BY 4.0 license (https://creativecommons.org/licenses/by/4.0/).

Submitted: 8 July 2021 Revised: 20 July 2021 Accepted: 31 August 2021 Published: 30 December 2021

We used correlation analysis to examine whether changes in grey matter volume in patients correlated with clinical presentation. gray matter volume was markedly reduced in neovascular glaucoma patients than healthy controls in the following brain regions: left cingulum anterior/medial frontal gyrus; left middle frontal gyrus, orbital part; left inferior frontal gyrus, orbital part; superior temporal gyrus/right frontal inferior orbital part. VBM directly suggests that neovascular glaucoma patients have changed in the volume of multiple brain regions. These changes exist in brain areas related to the visual pathway, as well as other brain areas which are not related to vision. The alteration of specific brain areas are closely related to clinical symptoms such as increased intraocular pressure and optic nerve atrophy in neovascular glaucoma patients. In conclusion, neovascular glaucoma may cause paralgesia, anxiety, and depression in patients.

\section{Keywords}

Voxel-based morphometry; Neovascular glaucoma; Fmri; Visual pathway

\section{Introduction}

Glaucoma is a leading cause of blindness globally [1], with neovascular glaucoma (NVG) being one of the most complicated forms of this illness. The primary pathological manifestations of patients with NVG include hyperplasia of intraocular fibrovascular tissue, obstruction of the trabecular meshwork, and peripheral anterior iris adhesion, which could lead to the obstruction of aqueous humor outflow and an increase in intraocular pressure [2] (Fig. 1). NVG can cause deficits in visual function as well as severe pain, both of which will affect quality of life [3]. Clinically, NVG is divided into three stages: Rubeosis, invasion of anterior chamber angle without closure and invasion of anterior chamber angle with closure. NVG is often secondary to diabetic retinopathy (DR), central retinal vein occlusion (CRVO), intravascular inflammation, and tumors $[2,4]$. The production of vascular endothelial growth factor (VEGF) secondary to retinal ischemia is the most common causes of NVG [5, 6]. VEGF [7, 8], Ephrin type-A re- ceptor 2 (EphA2) [9], platelet derived growth factor (PDGF) [10], hypoxia-inducible factor- $\alpha(\mathrm{HIF}-\alpha)$ [11], and fibroblast growth factors (FGFs) $[12,13]$ are all considered to be related to the onset of NVG.

Due to the complex etiology and pathogenesis of NVG, there are no precise indicators or guidelines which apply to the development and outcomes of this disease. Therefore, clinical treatment is complicated, and etiological therapies mainly included the elimination of retinal ischemia by photocoagular, cryotherapy and anti-VEGF therapy. Principles of clinical treatment include treatment of the primary diseases and timely elimination of retinal ischemia to control clinical symptoms. The core strategy of therapy involves reduction of intraocular pressure and retinal ischemic maculopathy, and eliminating neovascularization.

Magnetic resonance imaging (MRI) is well established in clinical work as a highly sensitive, non-invasive method of imaging soft tissues in the human body and observing their morphology and pathological changes. MRI offers advantages over X-ray or other imaging methods [14], and functional MRI (fMRI) allows voxel-based morphometry (VBM) for the analysis of brain morphology in fine spatial detail. VBM provides measurement of any changes in local gray matter volume (GMV), white matter volume (WMV) and the volume of the whole brain. Altered morphology in brain tissue may reflect the mechanism, development trend and clinical manifestations of disease, providing important information for practical clinical work. At present, in addition to cognitive diseases, VBM has been applied in ophthalmic diseases, including optic neuromyelitis [15], advanced monocular blindness [16], amblyopia [17], comitant strabismus [18], glaucoma [19], and acute eye pain [20] (Table 1, Ref. $[15,16,18,20-23])$.

Early studies have shown extensive and complex changes to cortical structure in glaucoma patients, and that these were significantly correlated with progression of the disease [24]. 


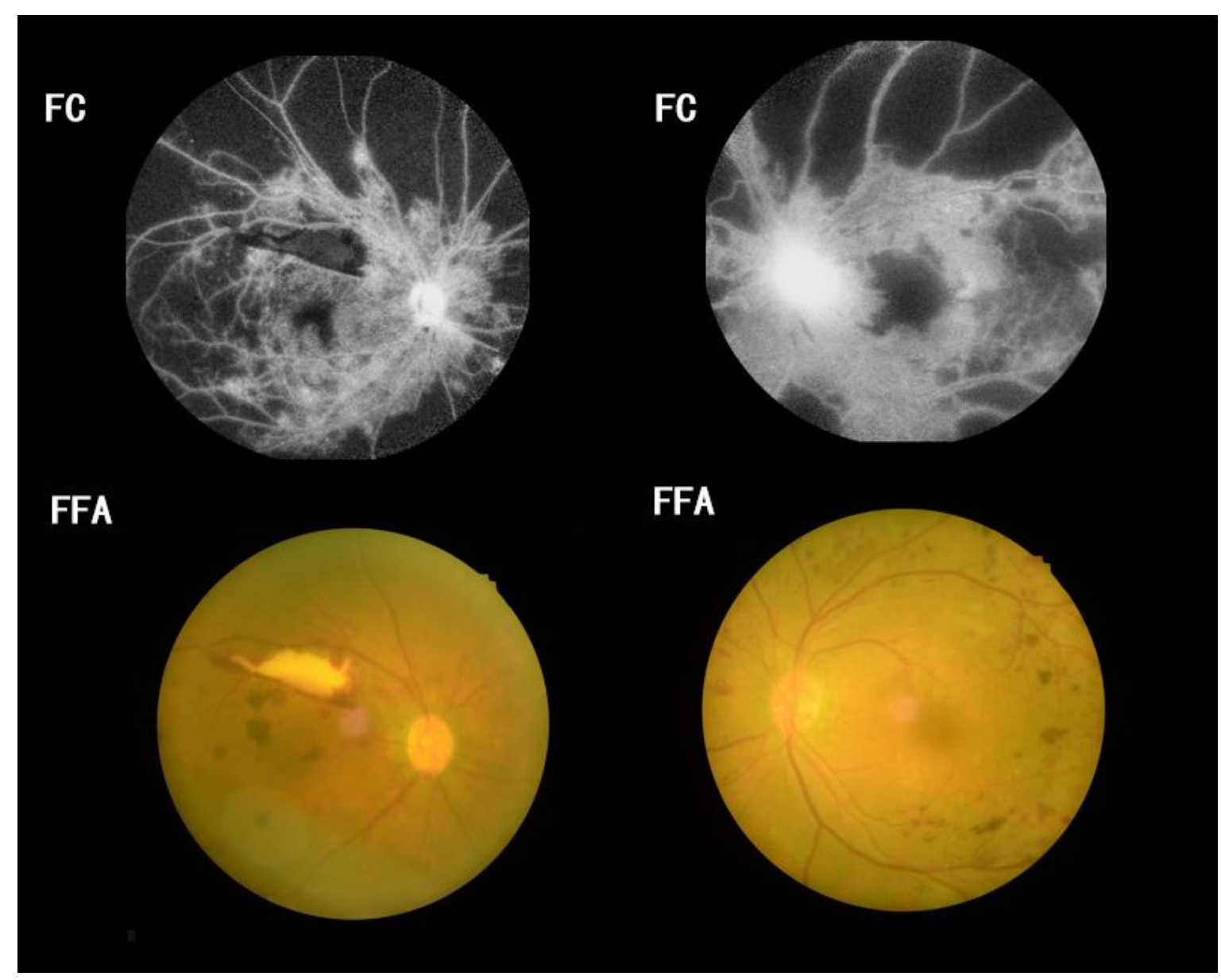

Fig. 1. Examples FC and FFA of NVG in a patient with ischemic central retinal vein occlusion. FC, fundus camera; FFA, fluorescence fundus angiography.

However, there is no grading system or other method for assessing changes in brain morphology in patients with NVG. VBM analysis was applied in the present research to explore whether there is a significant discrepancy of cortical volume between NVG patients and matched healthy controls (HCs), and to look for associations between those changes and the clinical manifestations and pathogenesis of NVG.

\section{Patients and methods}

Nineteen patients ( 9 males, 10 females) with bilateral NVG (secondary to ischemic central retinal vein occlusion) who had been diagnosed by the Ophthalmology Department of the First Affiliated Hospital of Nanchang University (Nanchang, China) were recruited to this research. Inclusion criteria for patients with NVG included: (1) slit lamp microscopy or gonioscopy examination shows neovascularization in the surface of iris and (or) anterior chamber angle; (2) intraocular pressure (IOP) $\geq 21 \mathrm{mmHg}$ (mean of at least three noncontact tonometry measurements); (3) retinal ischemia indicated by fundus fluorescein angiography.

The exclusion criteria for this group included: (1) the presence of acute keratitis, acute conjunctivitis or any other ocular inflammation (2) retinal ischemia caused by additional angiopathy (such as carotid stenosis); (3) a history of drugs addiction or alcoholism; (4) any condition contraindicating an MRI scan; (5) mental disorder, including but not limited to depression and bipolar disorder.

Nineteen individuals matched for age, gender and educational level met the above exclusion criteria and had no abnormal brain structure, function or metabolism evident on MRI examination.

The Medical Ethics Committee of the First Affiliated Hospital of Nanchang University (Nanchang, China) gave their approval to the study. The study design was conducted in accordance with the Declaration of Helsinki. Prior to participant signed an informed consent declaration.

\subsection{Structural MRI parameters}

All subjects underwent scanning on a 3-T MRI scanner (Siemens, Erlangen, Germany) with an 8-channel head coil. The fast gradient echo sequence using magnetization prepared rapid acquisition with gradient echo (MP-RAGE) was acquired to ensure high-resolution T1-weighted images covering the entire brain. The following were the specific parameters: repetition time (TR)/echo time (TE): 1900/2.26 ms; gap, $0.5 \mathrm{~mm}$; acquisition matrix: $256 \times 256$; field of 
Table 1. VBM used in ophthalmic disease.

\begin{tabular}{|c|c|c|c|c|}
\hline Author & Year & Disease & Brain region with altered GMV & Brain regions with altered WHV \\
\hline Huang et al. [15] & 2016 & Optic neuritis & $\begin{array}{l}\text { PoCG.L，IFG.L，ACG.L， MFG.R, } \\
\text { IPL.R }\end{array}$ & $\begin{array}{l}\text { MFG.R, SFG.R, PreCG.L, IPL.R, } \\
\text { FFG.L, IPL.L }\end{array}$ \\
\hline Shi et al. [16] & 2019 & Advanced monocular blindness & INS.L, INS.R, ACG.R & / \\
\hline OuYang et al. [18] & 2017 & Comitant strabismus & $\begin{array}{l}\text { TPOmid.L, LC, PCG.R, CUN.L, } \\
\text { PMC.R. }\end{array}$ & $\begin{array}{l}\text { TPOmid.L, TPOmid.R, PCUN.L, } \\
\text { PMC.R. }\end{array}$ \\
\hline Lan et al. [20] & 2019 & Acute eye pain & $\begin{array}{l}\text { CPL.L, Limbic Lobe-L,INS.R, INS.L, } \\
\text { THA.L, CAU.L, CUN.R }\end{array}$ & Whole brain \\
\hline Li et al. [21] & 2012 & $\begin{array}{l}\text { primary open-angle glaucoma, } \\
\text { POAG }\end{array}$ & $\begin{array}{l}\text { BA17, BA18, BA5, BA6, BA9, BA20, } \\
\text { BA39,BA7,BA21,BA22 }\end{array}$ & / \\
\hline Chan et al. [22] & 2004 & amblyopia & OEF, PEF, FEF, SEF, PFC & I \\
\hline Li et al. [23] & 2020 & Retinal detachment & $\begin{array}{l}\text { ACG.R, STG.R, ACG.R, CUN.R, } \\
\text { HIP.L, TPOmid.L }\end{array}$ & / \\
\hline
\end{tabular}

GMV, gray matter volume; WHV, white matter volume; TPOmid.L, left temporal pole: middle temporal gyrus; LC, left cerebellum; PCG.R, right posterior cingulate gyrus; CUN.L, left cuneus; PMC.R, right promotor cortex; PCUN.L, left precuneus; PoCG.L, left postcentral gyrus; IFG.L, left inferior frontal gyrus; ACG.L, left anterior cingulate gyrus; MFG.R, right middle frontal gyrus; IPL.R, right inferior parietal lobule; SFG.R, right superior frontal gyrus; PreCG.L, left precentral gyrus; FFG.L, left fusiform gyrus; INS.R, right insular lobe; INS.L, left insular lobe; STG.R, right superior temporal gyrus; HIP.L, left hippocampus; CPL.L, left cerebellum posterior lobe; INS.R, right insula; INS.L, left insula; THA.L, left thalamus; ACU.L, left caudate; CUN.R, right cuneus; OEF, occipital eye field; PEF, parietal eye field; SEF, supplementary eye field; PFC, prefrontal cortex; BA, brodmann area.

view: $250 \times 250 \mathrm{~mm}$; flip angle: $9^{\circ} ; 1.0 \mathrm{~mm}$ thickness and 176 slices. A neuroradiologist evaluated all images and no cerebral parenchymal abnormality was found in any of the subjects.

\subsection{Image processing}

To optimize data quality, MRIcro software (Toshiba Inc., Innovista. www.MRIcro.com) was utilized to filter out irrelevant information. Matlab 7.9.0 (R2009b; The Mathsworks Inc., Natick, MA, USA) was used to run the voxel-based morphometry toolbox (VBM8, Wellcome Trust Centre for Neuroimaging, London, UK. http://dbm.neuro.uni-jena.de /vbm8/) within statistical parametric mapping (SPM version 8, Wellcome Trust Centre for Neuroimaging, London, UK. http://www.fil.ion.ucl.ac.uk/spm). VBM8 was set to partition the entire brain into cerebral gray matter, white matter, and cerebrospinal fluid as the default estimation option. Due to individual variability in brain structure, this study used the Diffeomorphic Anatomical Registration through Exponentiated Lie Algebra (DARTEL) method (implemented in VBM8) was being used to normalize images to the Montreal Institute of Neurology (MNI) standardized space, and to create universal templates for gray matter and white matter. The templates were used to standardize the gray matter and white matter of all subjects. To minimize noise in the segmented image and improve the image signal-to-noise ratio and quality, a $6 \mathrm{~mm}$ full width half maximum Gaussian kernel was used to smooth the modulation volume. Finally, for analysis, smoothed images were segregated into subject groups.

\subsection{Data processing}

In the SPM8 toolbox (http://www.fil.ion.ucl.ac.uk/spm), a general linear model (GLM) was employed. GMV was compared between groups using an independent sample $\mathrm{t}$ test $(\alpha=0.05)$ after accounting for age, gender and education level using the Resting-State fMRI Data Analysis Toolkit (REST) (http://www.restfmri.net). False discovery rate was used to correct for multiple comparisons. For providing a color map, significant statistical voxels were superimposed on 3D T1-weighted standardized images. Twenty adjacent voxels (threshold $=0.01$ ) were selected to examine GMV differences between groups. The analysis of mean GMV values of discrete brain regions was performed using receiver operating characteristic (ROC) curves to assess diagnostic value.

Brain regions with significant GMV value were identified as regions of interest (ROI) using REST software. The mean GMV of all voxels in each region was considered the GMV value of the ROI. Correlation analysis was used to look for any relationship between abnormal GMV values in the NVG group and their clinical features.

The hospital anxiety and depression scale (HADS), which patients completed and graded, was used to assess the level of anxiety and depression in NVG patients [25]. In the NVG group, the Visual Analogue Score (VAS) was used to assess pain perception. GraphPad prism 7 (GraphPad Software Inc., San Diego, CA, USA) was the tool we used to diagnose the relationship between HADS, VAS and GMV values, respectively.

Basic clinical data (duration of disease, IOP and best corrected visual acuity) of NVG patients and HCs were collected. SPSS 20.0 (SPSS Inc., Chicago, IL, USA) was adopted to perform independent sample $t$-tests comparing clinical and demographic variables between the two groups.

$p$ values of less than 0.05 were deemed significant in all statistical analyses. 


\section{Result}

\subsection{Participants}

Between the NVG and HC groups, there was no significant difference in age $(p=0.953)$ or handedness $(p>0.99)$. A significant difference between groups was found, however, in monocular best-corrected visual acuity of the left $(p=0.021)$ and right eye $(p=0.026)$. IOP was also significantly different between groups in both eyes (left eye $p=0.007$; right eye $p=$ 0.011). More details are shown in Table 2.

Table 2. Demographics and behavioral results of all participants.

\begin{tabular}{lcccc}
\multicolumn{5}{c}{ participants. } \\
\hline NVG & HC & t-value & $p$-value \\
\hline Male/female & $9 / 10$ & $9 / 10$ & N/A & $>0.99$ \\
Age (years) & $55.12 \pm 10.26$ & $54.95 \pm 9.12$ & 0.423 & 0.953 \\
Handedness & $19 \mathrm{R}$ & $19 \mathrm{R}$ & $\mathrm{N} / \mathrm{A}$ & $>0.99$ \\
Duration (years) & $4.16 \pm 1.41$ & $\mathrm{~N} / \mathrm{A}$ & $\mathrm{N} / \mathrm{A}$ & $\mathrm{N} / \mathrm{A}$ \\
Best-corrected VA-L & $0.25 \pm 0.10$ & $1.05 \pm 0.15$ & 5.764 & 0.021 \\
Best-corrected VA-R & $0.25 \pm 0.05$ & $0.95 \pm 0.10$ & 5.131 & 0.026 \\
OP-L & $28.12 \pm 11.35$ & $14.67 \pm 4.75$ & 12.842 & 0.007 \\
IOP-R & $27.12 \pm 13.38$ & $15.16 \pm 3.33$ & 11.529 & 0.011 \\
\hline
\end{tabular}

Independent $t$-tests ( $p<0.05$ represented statistically significant differences) and chi-squared test were adopted to verify the significance of difference in age, male/female and handedness, respectively. NVG, neovascular glaucoma; HCs, healthy controls; N/A, not applicable; VA, visual acuity; IOP, intraocular pressure; L, left eye; R, right eye.

\subsection{VBM differences between groups}

In the accompanying brain regions, the VBM value in the NVG group was vastly smaller than HCs: left cingulum anterior/medial frontal gyrus $(t=6.2185, p<0.001)$; left middle frontal gyrus, orbital part $(\mathrm{t}=5.6938, p<0.001)$; left inferior frontal gyrus, orbital part $(t=7.0916, p<0.001)$; right superior temporal gyrus/right frontal inferior orbital part $(\mathrm{t}$ $=7.3406, p<0.001)($ Table 3 , Fig. 2).

\subsection{Correlation analyses}

In NGV patients, GMV in the left middle frontal gyrus, orbital part was negatively correlated with HADS $(\mathrm{r}=-0.936$, $p<0.001)$ and VAS $(\mathrm{r}=-0.872, p<0.001)$ (Fig. 3).

\subsection{ROC curve}

The areas under the ROC curves were 0.871 for left Cingulum anterior/Medial Frontal Gyrus, 0.885 for left middle frontal gyrus, orbital part, 0.941 for left inferior frontal gyrus, orbital part, and 0.926 for right superior temporal gyrus/right frontal inferior orbital part (Fig. 4).

\section{Discussion}

This is the first study of using VBM to assess gray matter volume abnormalities in NVG patients. VBM has, however, been widely used in research on both neurological and ophthalmological diseases, and has shown clear advantages in the exploration of the pathological mechanism of these diseases.
Studies have established that glaucoma patients generally show atrophy of specific brain areas or changes in functional connectivity between brain areas. Aditya et al. [19] showed varying degrees of volume reduction along the visual pathway in glaucoma. Laura et al. [26] showed reduced cortical thickness and volume in both primary visual cortex and association visual areas in glaucoma patients. Fukuda et al. [27] found decreased gray matter density (GMD) in primary open-angle glaucoma (POAG) patients, particularly in the visual cortical areas (Brodmann areas 17, 18, 19). In patients with POAG, Jiang et al. [28] found altered volume in brain regions which are highly correlated with the retinal nerve fiber layer. Similarly, Ziko et al. [29] found that VBM showed significantly decreased volume in the visual pathway and other brain areas which are related with the visual system. Li et al. [21] suggested that POAG patients with advanced disease show degenerative changes in visual-related cerebral cortex, dorsal and ventral visual pathways.

In this study, the NVG group experienced statistically significant GMV reductions when compared to the HCs in left cingulum anterior/medial frontal gyrus; left middle frontal gyrus, orbital part; and the right temporal: superior temporal gyrus/right frontal inferior orbital part.

The cingulate gyrus occupies a crucial position in the limbic system [30] and exerts an important role in emotional control and pain management. According to current studies, the anterior cingulate gyrus is involved in not only the regulation of heart rate or blood pressure, and other autonomic nerve activities [31]. But it's also implicated in a variety of sophisticated body and visceral motor processes, as well as pain responses, attention maintenance [32], decisionmaking processes [33], and pain management [34]. Previous studies have demonstrated that the anterior cingulate gyrus cortex of the right dorsal horn may be related to visual cognition and emotion [35]. Results of the present study indicate decreased gray matter volume of anterior cingulate gyrus in NVG patients. This result suggesting that NVG patients may have dysfunction in visual cognitive function [22].

The frontal gyrus is an important component of the dorsal visual pathway [36]. It is concerned with recognition and judgement based on spatial information about objects and simultaneously plays an important role in optic nerve positioning, multi-retina correspondence, and the formation of scotoma of the fixation point [37]. The frontal gyrus includes superior, medial and inferior frontal gyrus, and abnormalities of its structure or function have been investigated in many ophthalmic diseases, including but not limited to diabetic retinopathy [38], strabismus with amblyopia [39], corneal ulcer [40] and ophthalmectomy [14]. In this study, we found that VBM results indicated significant GMV reduction in all of these regions (medial, superior, and inferior frontal gyrus), which are the same as Wang et al. [41] and it may be related to increased intraocular pressure (IOP). 
Table 3. GMV discrepancies between the NVG group and the HCs in several brain areas.

\begin{tabular}{llcccc}
\hline \multirow{2}{*}{ GMV } & \multicolumn{4}{c}{ NVG group and HCs } & \multicolumn{3}{c}{ MNI coordinates } \\
\cline { 2 - 6 } & Brain areas & Peak t values & X & Y & Z \\
\hline NVG $<$ HCs & & & & & \\
1. & Temporal pole sup R/Frontal inf orb R & 7.3406 & 34.5 & 13.5 & -25.5 \\
2. & Frontal inf orb L & 7.0916 & -28.5 & 7.5 & -22.5 \\
3. & Frontal mid orb L & 5.6938 & -40.5 & 43.5 & -9 \\
4. & Cingulum ant L/Medial frontal gyrus & 6.2185 & 0 & 37.5 & 19.5 \\
\hline
\end{tabular}

GMV, gray matter volume; NVG, neovascular glaucoma; HCs, health controls; MNI, montreal nroradiology neurological institute.

A

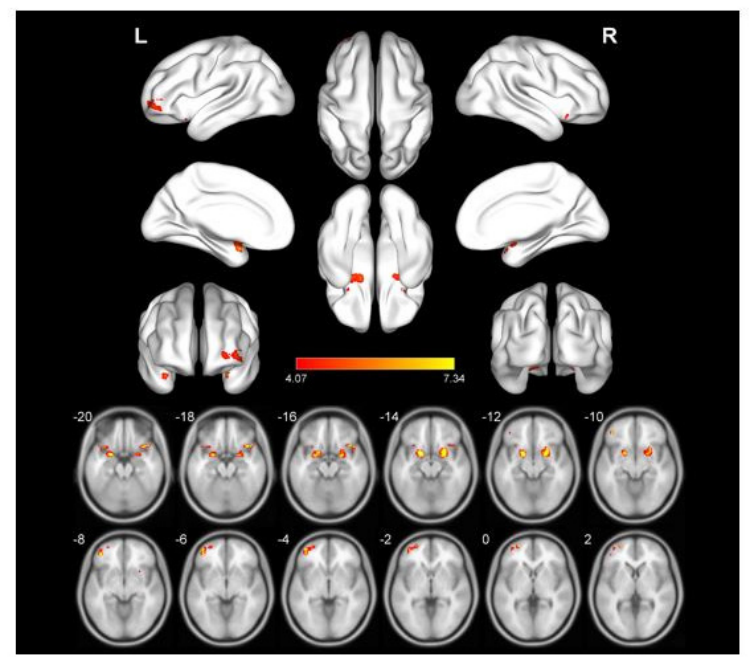

B

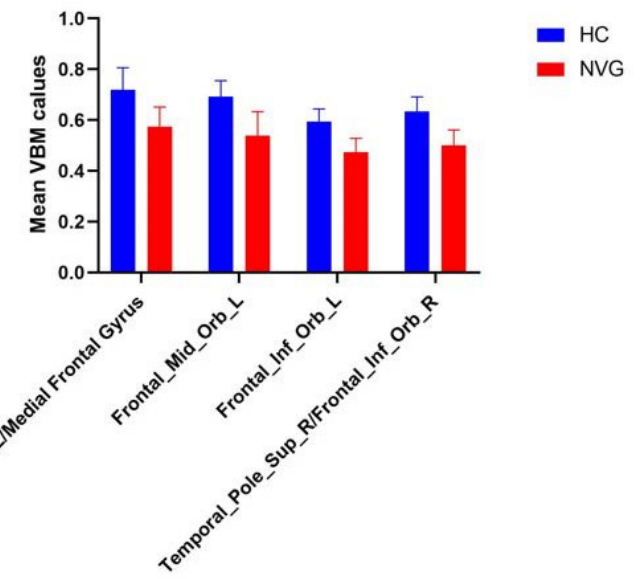

Brain regions with altered VBM values

Fig. 2. Spontaneous brain activity in NVG patients. The two groups of brain regions show statistically significant differences in terms of VBM. (A) Patients presented alternated VBM valued in Cingulum Ant L/Medial Frontal Gyrus, Frontal Mid Orb L, Frontal Inf Orb L, Temporal Pole Sup R/Frontal Inf Orb R compared with that reported in the HC group, the red areas indicate the variable VBM values. (B) The mean GMV values of two groups. VBM, voxel-based morphometry; NVG, neovascular glaucoma; Cingulum Ant L, left anterior cingulum; Frontal Mid Orb L, left middle frontal gyrus, orbital part; Frontal Inf Orb L, left inferior frontal gyrus, orbital part; Temporal Pole Sup R, right temporal: superior temporal gyrus; Frontal Inf Orb R, right frontal inferior orbital part; HC, healthy controls.

A

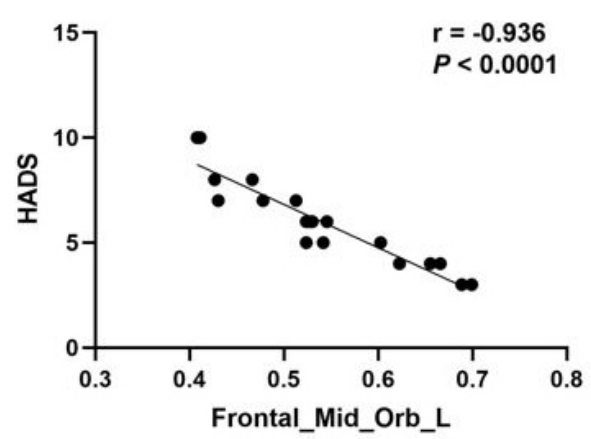

B

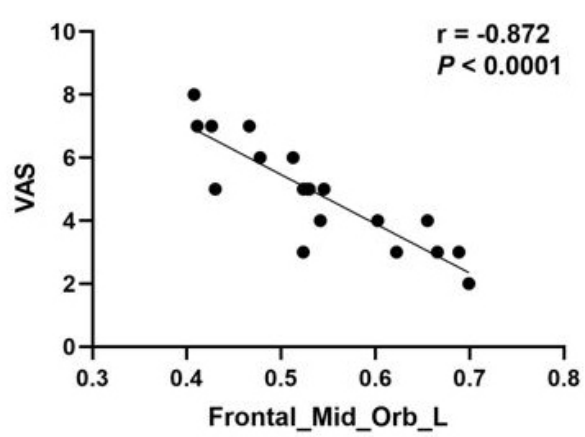

Fig. 3. Correlation analysis between the score of HADS and the GMV values of the Frontal Mid Orb L. (A) GMV values showed a negative correlation with the scores of HADS ( $\mathrm{r}=-0.936, p<0.0001$ ). (B) GMV values showed a negative correlation with the scores of VAS ( $\mathrm{r}=-0.872, p<-0.0001$ ). HADS, hospital anxiety and depression scale; VAS, Visual Analogue Score; GMV, gray matter volume. Frontal Mid Orb L, middle frontal gyrus, orbital part.

The medial frontal gyrus is the medial side of the superior frontal gyrus, located anterior to the parasitical central lobule. Research suggests that the medial frontal gyrus is linked to the decision-making process and the subsequent execution process [42]. In addition, the frontal eye field (FEF) area in the center of the medial frontal gyrus regulates spontaneous eye movements and is also linked to emotional transfer [43]. The cortex of the medial frontal gyrus represents one 
Table 4. Changed FCD values in brain regions and its potential impact.

\begin{tabular}{lll}
\hline Brain region & Brain function & Anticipated results \\
\hline Cingulum anterior gyrus & Cognition; emotion; memory and other cognitive functions & $\begin{array}{l}\text { Defects of cognition and emotion; abnormal pain man- } \\
\text { agement }\end{array}$ \\
Medial Frontal Gyrus & Involving in default mode network (DMN); & $\begin{array}{l}\text { Dysfunction of DMN; anxiety and depression; pain- } \\
\text { related diseases }\end{array}$ \\
Middle frontal gyrus & Consist of Frontal eye field; language processing; part of DMN & $\begin{array}{l}\text { Depression and anxiety; impairment of eye motion and } \\
\text { visual acuity; increasing pain sensitivity }\end{array}$ \\
Inferior frontal gyrus & Emotional and cognitive empathy; part of DMN & $\begin{array}{l}\text { Impairment of ocular movement and cognitive; depres- } \\
\text { sion and anxiety }\end{array}$ \\
Superior temporal gyrus & $\begin{array}{l}\text { Relating to visual information processing and visual search in- } \\
\text { sight, affecting auditory processing and auditory memory }\end{array}$ & $\begin{array}{l}\text { Abnormal visual information processing and visual im- } \\
\text { pairment }\end{array}$
\end{tabular}

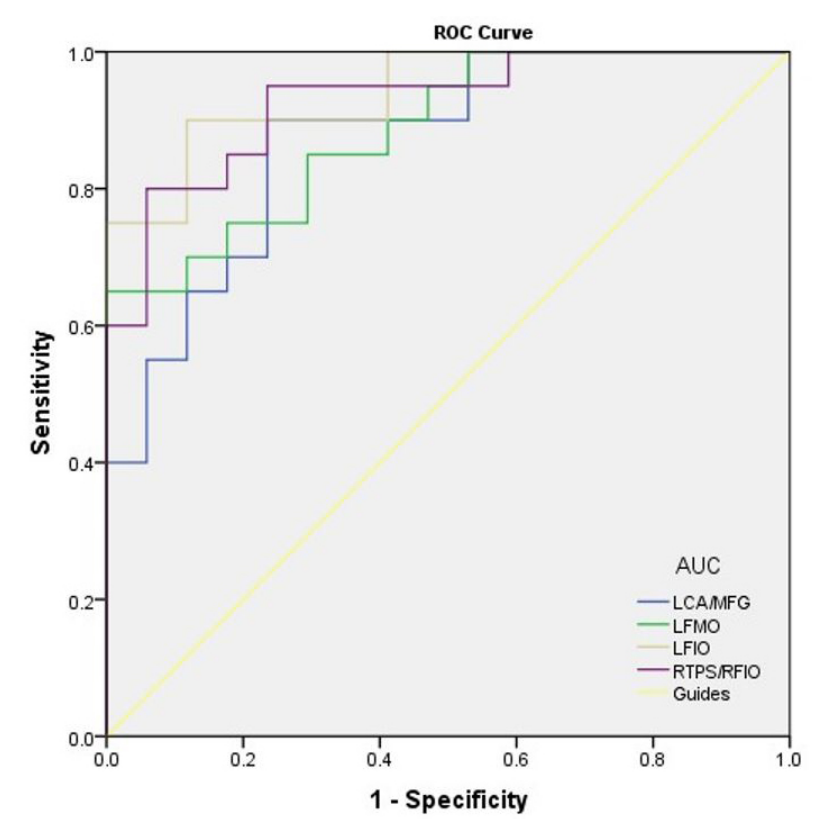

Fig. 4. ROC curve of the VBM values in altered brain areas. The area under the ROC curve were $0.871(p<0.0001$; 95\% CI: 0.758-0.984) for LCA/MFG, LFMO 0.885 ( $p<0.0001$; 95\% CI: 0.783-0.988), LFIO 0.941 $(p<0.0001$; 95\% CI: 0.871-1.000), RTPS/RFIO 0.926 ( $p<0.0001 ; 95 \% \mathrm{CI}$ : 0.845-1.000). AUC, area under the curve; ROC, receiver operating characteristic; CI, confidence interval; LCA/MFG, left anterior Cingulum/Medial Frontal Gyrus; LFMO, left middle frontal gyrus, orbital part; LFIO, left inferior frontal gyrus, orbital part; RTPS/RFIO, right temporal pole: superior temporal gyrus/right inferior frontal gyrus, orbital part.

of the components of the default model network which includes the inferior parietal cortex, medial and superior frontal gyrus and precuneus [44, 45]. Furthermore, it plays a fundamental role in specific emotion such as depression and anxiety [46]. The GMV of the medial frontal gyrus was diminished in this study, illustrating that patients with NVG may have visual pathways that are compromised. Jiang et al. [28] found that the frontal gyrus area of patients with primary open-angle glaucoma (POAG) shows increased volume and blood oxygenation level dependent (BOLD ) signals. They concluded that this may be due to edema of local cerebral tissue and microsomal activation, that increased blood flow may be a pathological compensatory response after structural and functional impairment, and that these results may indicate nerve damage in the frontal gyrus. This hypothesis may explain why Williams et al. [47] found increased medial frontal gyrus volume in glaucoma patients in early stage, which is contrary to this study.

The decrease in GMV volume in the superior and inferior frontal gyrus in the present study agrees with the results of other existing studies [21,48], supporting the finding of damaged cortical visual pathways in NVG patients. These results collectively suggest that NVG patients may have abnormalities in eye movement, cognitive function and visual processing ability.

The superior temporal gyrus is involved in visual signal processing, auditory processing, auditory memory and other physiological processes. The superior and middle temporal gyri are thought to specialize in processing dorsal signals along the visual pathway [49]. Previous studies have observed abnormalities in the superior temporal gyrus in specific ophthalmopathy, including but not limited to optic neuritis [50], diabetic retinopathy [51], comitant strabismus [18], and retinal detachment [23]. In this study, the decrease in GMV in the superior temporal gyrus may indicate atrophy of temporal retinal fibers in NVG patients [29] (Table 4).

Notably, some of the studies also mentioned that betaamyloid accumulation may be associated with altered GMV in NVG patients [52], which happens to be a typical pathological change in Alzheimer's patients. There is still a plenty of room to be investigated.

The present study has the following limitations: (1) The participants included in this study may be insufficient. However, selection followed precise inclusion and exclusion criteria, and the differences between potentially confounding factors were not statistically significant; (2) The study samples are all derived from hospital outpatient data, which risks selection bias; (3) Due to equipment and technical limitations, the spatial resolution of imaging may not be optimal.

\section{Conclusions}

In summary, NVG patients show decreased GMV values in certain brain areas, the dysfunction of those brain areas 
may be related to the appearance of corresponding symptom in NVG patients such as the depression and anxiety.

\section{Author contributions}

Conceptualization, methodology, writing original draft preparation, writing reviewing and editing-HZ, YDS, RBL. Data curation, formal analysis-QMG, YCP, LJZ, HYS, QYL. Resources, methodology, supervision, projection administration, funding acquisition-YS.

\section{Ethics approval and consent to participate}

All research methods were approved by the committee of the medical ethics of the First Affiliated Hospital of Nanchang University (2015016) and were in accordance with the 1964 Helsinki declaration and its later amendments or comparable ethical standards. All subjects were explained the purpose, method, potential risks and signed an informed consent form.

\section{Acknowledgment \\ Not applicable.}

\section{Funding}

This research was funded by National Natural Science Foundation of China, grant number 81660158, 81460092, and 81400372; Natural Science Key Project of Jiangxi Province, grant number 20161ACB21017; Health Development Planning Commission Science Foundation of Jiangxi Province, grant number 20175116.

\section{Conflict of interest}

The authors declare no conflict of interest.

\section{References}

[1] Leske MC. Open-angle glaucoma-an epidemiologic overview. Ophthalmic Epidemiology. 2007; 14: 166-172.

[2] Kwon J, Jee D, La TY. Neovascular glaucoma after vitrectomy in patients with proliferative diabetic retinopathy. Medicine. 2017; 96: e6263.

[3] Seymenoğlu RG, Ulusoy MO, Başer EF. Safety and efficacy of panretinal photocoagulation in patients with high-risk proliferative diabetic retinopathy using pattern scan laser versus conventional YAG laser. Journal of Medical Sciences. 2016; 32: 22-26.

[4] Sun Y, Liang Y, Zhou P, Wu H, Hou X, Ren Z, et al. Anti-VEGF treatment is the key strategy for neovascular glaucoma management in the short term. BMC Ophthalmology. 2016; 16: 150.

[5] Mathew R, Barton K. Anti-Vascular Endothelial Growth Factor Therapy in Glaucoma Filtration Surgery. American Journal of Ophthalmology. 2011; 152: 10-15.

[6] Bochmann F, Kaufmann C, Becht CN, Guber I, Kaiser M, Bachmann LM, et al. ISRCTN12125882-Influence of topical antiVEGF (Ranibizumab) on the outcome of filtration surgery for glaucoma-Study Protocol. BMC Ophthalmology. 2011; 11: 1.

[7] Ma KT, Yang JY, Kim JH, Kim NR, Hong S, Lee ES, et al. Surgical Results of Ahmed Valve Implantation with Intraoperative Bevacizumab Injection in Patients with Neovascular Glaucoma. Journal of Glaucoma. 2012; 21: 331-336.

[8] Kwong TQ, Mohamed M. Anti-vascular endothelial growth factor therapies in ophthalmology: current use, controversies and the future. British Journal of Clinical Pharmacology. 2014; 78: 699706.
[9] Li R, Yuan W, Mei W, Yang K, Chen Z. MicroRNA 520d-3p inhibits gastric cancer cell proliferation, migration, and invasion by downregulating EphA2 expression. Molecular and Cellular Biochemistry. 2014; 396: 295-305.

[10] Crawford T, Alfaro III D, Kerrison J, Jablon E. Diabetic Retinopathy and Angiogenesis. Current Diabetes Reviews. 2009; 5: 8-13.

[11] Lin M, Chen Y, Jin J, Hu Y, Zhou K K, Zhu M, et al. Ischaemiainduced retinal neovascularisation and diabetic retinopathy in mice with conditional knockout of hypoxia-inducible factor-1 in retinal Muller cells. Diabetologia. 2011, 54: 1554-1566.

[12] Ornitz DM, Itoh N. The Fibroblast Growth Factor signaling pathway. Wiley Interdisciplinary Reviews Developmental Biology. 2015; 4: 215-266.

[13] Beenken A, Mohammadi M. The FGF family: biology, pathophysiology and therapy. Nature Reviews Drug Discovery. 2009; 8: 235-253.

[14] Zhang B. Altered spontaneous brain activity pattern in patients with ophthalmectomy: a resting-state fMRI study. International Journal of Ophthalmology. 2020; 13: 263-270.

[15] Huang X, Zhang Q, Hu P H, Zhong Y L, Zhang Y, Wei R, et al. White and Gray Matter Volume Changes and Correlation with Visual Evoked Potential in Patients with Optic Neuritis: A VoxelBased Morphometry Study. Medical Science Monitor. 2016; 22: 1115-1123.

[16] Shi W, He Y, Li Q, Tang L, Li B, Lin Q, et al. Central network changes in patients with advanced monocular blindness: a voxelbased morphometric study. Brain and Behavior. 2019; 9: e01421.

[17] Barnes GR, Li X, Thompson B, Singh KD, Dumoulin SO, Hess RF. Decreased gray matter concentration in the lateral geniculate nuclei in human amblyopes. Investigative Ophthalmology \& Visual Science. 2010; 51: 1432-1438.

[18] Ouyang J, Yang L, Huang X, Zhong Y, Hu P, Zhang Y, et al. The atrophy of white and gray matter volume in patients with comitant strabismus: Evidence from a voxel-based morphometry study. Molecular Medicine Reports. 2017; 16: 3276-3282.

[19] Hernowo AT, Boucard CC, Jansonius NM, Hooymans JMM, Cornelissen FW. Automated morphometry of the visual pathway in primary open-angle glaucoma. Investigative Ophthalmology \& Visual Science. 2011; 52: 2758-2766.

[20] Lan D, Zhu P, He Y, Xu Q, Su T, Li B, et al. Gray Matter Volume Changes in Patients with Acute Eye Pain: a Voxel-Based Morphometry Study. Translational Vision Science \& Technology. 2019; 8: 1.

[21] Li C, Cai P, Shi L, Lin Y, Zhang J, Liu S, et al. Voxel-based Morphometry of the Visual-related Cortex in Primary Open Angle Glaucoma. Current Eye Research. 2012; 37: 794-802.

[22] Chan ST, Tang KW, Lam KC, Chan LK, Mendola JD, Kwong KK. Neuroanatomy of adult strabismus: a voxel-based morphometric analysis of magnetic resonance structural scans. Neuroimage. 2004; 22: 986-994.

[23] Li B, Liu Y, Li H, Yuan Q, Zhu P, Ye L, et al. Reduced gray matter volume in patients with retinal detachment: evidence from a voxel-based morphometry study. Acta Radiologica. 2020; 61: 395403.

[24] Dai H, Yin D, Hu C, Morelli JN, Hu S, Yan X, et al. Whole-brain voxel-based analysis of diffusion tensor MRI parameters in patients with primary open angle glaucoma and correlation with clinical glaucoma stage. Neuroradiology. 2013; 55: 233-243.

[25] Djukanovic I, Carlsson J, Årestedt K. Is the Hospital Anxiety and Depression Scale (HADS) a valid measure in a general population 65-80 years old? A psychometric evaluation study. Health and Quality of Life Outcomes. 2017; 15: 193.

[26] Nuzzi R, Dallorto L, Rolle T. Changes of Visual Pathway and Brain Connectivity in Glaucoma: a Systematic Review. Frontiers in Neuroscience. 2018; 12: 363.

[27] Fukuda M, Omodaka K, Tatewaki Y, Himori N, Matsudaira I, Nishiguchi KM, et al. Quantitative MRI evaluation of glaucomatous changes in the visual pathway. PLoS ONE. 2018; 13: e0197027. 
[28] Jiang M, Zhou Q, Liu X, Shi C, Chen J, Huang X. Structural and functional brain changes in early- and mid-stage primary openangle glaucoma using voxel-based morphometry and functional magnetic resonance imaging. Medicine. 2017; 96: e6139.

[29] Zikou AK, Kitsos G, Tzarouchi LC, Astrakas L, Alexiou GA, Argyropoulou MI. Voxel-based morphometry and diffusion tensor imaging of the optic pathway in primary open-angle glaucoma: a preliminary study. American Journal of Neuroradiology. 2012; 33: 128-134.

[30] Delvecchio G, Rossetti MG, Caletti E, Arighi A, Galimberti D, Basilico P, et al. The Neuroanatomy of Somatoform Disorders: a Magnetic Resonance Imaging Study. Psychosomatics. 2019; 60: 278-288.

[31] Kimmerly DS. A review of human neuroimaging investigations involved with central autonomic regulation of baroreflexmediated cardiovascular control. Autonomic Neuroscience. 2017; 207: 10-21.

[32] Bryden DW, Johnson EE, Tobia SC, Kashtelyan V, Roesch MR. Attention for learning signals in anterior cingulate cortex. Society for Neuroscience. 2011; 31: 18266-18274.

[33] Blanchard TC, Strait CE, Hayden BY. Ramping ensemble activity in dorsal anterior cingulate neurons during persistent commitment to a decision. Journal of Neurophysiology. 2015; 114: 2439_ 2449.

[34] Urien L, Xiao Z, Dale J, Bauer EP, Chen Z, Wang J. Rate and Temporal Coding Mechanisms in the Anterior Cingulate Cortex for Pain Anticipation. Scientific Reports. 2018; 8: 8298.

[35] Shinoura N, Yamada R, Tabei Y, Shiode T, Itoi C, Saito S, et al. The right dorsal anterior cingulate cortex may play a role in anxiety disorder and visual function. Neurological Research. 2013; 35: 6570.

[36] Maunsell J, Nealey T, DePriest D. Magnocellular and parvocellular contributions to responses in the middle temporal visual area (MT) of the macaque monkey. Journal of Neuroscience. 1990; 10: 3323-3334.

[37] Johnson PB, Ferraina S, Bianchi L, Caminiti R. Cortical networks for visual reaching: physiological and anatomical organization of frontal and parietal lobe arm regions. Cerebral Cortex. 1996; 6: 102-119.

[38] Wang Y, Shao Y, Shi W, Jiang L, Wang X, Zhu P, et al. The predictive potential of altered spontaneous brain activity patterns in diabetic retinopathy and nephropathy. EPMA Journal. 2019; 10: 249-259.

[39] Min Y, Su T, Shu Y, Liu W, Chen L, Shi W, et al. Altered spontaneous brain activity patterns in strabismus with amblyopia patients using amplitude of low-frequency fluctuation: a restingstate fMRI study. Neuropsychiatric Disease and Treatment. 2018; 14: 2351-2359.

[40] Zhu F, Tang L, Zhu P, Lin Q, Yuan Q, Shi W, et al. Restingstate functional magnetic resonance imaging (fMRI) and functional connectivity density mapping in patients with corneal ulcer. Neuropsychiatric Disease and Treatment. 2019; 15: 1833-1844.
[41] Wang Y, Wang X, Zhou J, Qiu J, Yan T, Xie Y, et al. Brain morphological alterations of cerebral cortex and subcortical nuclei in high-tension glaucoma brain and its associations with intraocular pressure. Neuroradiology. 2020; 62: 495-502.

[42] Talati A, Hirsch J. Functional Specialization within the Medial Frontal Gyrus for Perceptual Go/no-Go Decisions Based on "what," "when," and "where” Related Information: an fMRI Study. Journal of Cognitive Neuroscience. 2005; 17: 981-993.

[43] Bruce CJ, Goldberg ME, Bushnell MC, Stanton GB. Primate frontal eye fields. II. Physiological and anatomical correlates of electrically evoked eye movements. Journal of Neurophysiology. 1985; 54: 714-734.

[44] Zald DH, McHugo M, Ray KL, Glahn DC, Eickhoff SB, Laird AR. Meta-Analytic Connectivity Modeling Reveals Differential Functional Connectivity of the Medial and Lateral Orbitofrontal Cortex. Cerebral Cortex. 2014; 24: 232-248.

[45] Liu Y, Li L, Li B, Feng N, Li L, Zhang X, et al. Decreased Triple Network Connectivity in Patients with Recent Onset PostTraumatic Stress Disorder after a Single Prolonged Trauma Exposure. Scientific Reports. 2017; 7: 12625.

[46] Pa J, Possin KL, Wilson SM, Quitania LC, Kramer JH, Boxer AL, et al. Gray matter correlates of set-shifting among neurodegenerative disease, mild cognitive impairment, and healthy older adults. International Neuropsychological Society. 2010; 16: 640-650.

[47] Williams AL, Lackey J, Wizov SS, Chia TMT, Gatla S, Moster ML, et al. Evidence for widespread structural brain changes in glaucoma: a preliminary voxel-based MRI study. Investigative Ophthalmology \& Visual Science. 2013; 54: 5880-5887.

[48] Chen WW, Wang N, Cai S, Fang Z, Yu M, Wu Q, et al. Structural Brain Abnormalities in Patients with Primary Open-Angle Glaucoma: a Study with 3T MR Imaging. Investigative Opthalmology \& Visual Science. 2013; 54: 545-554.

[49] Ungerleider LG, Haxby JV. "what" and "where" in the human brain. Current Opinion in Neurobiology. 1994; 4: 157-165.

[50] Werring DJ, Bullmore ET, Toosy AT, Miller DH, Barker GJ, MacManus DG, et al. Recovery from optic neuritis is associated with a change in the distribution of cerebral response to visual stimulation: a functional magnetic resonance imaging study. Journal of Neurology, Neurosurgery and Psychiatry. 2000; 68: 441-449.

[51] Wang Z, Zou L, Lu Z, Xie X, Jia Z, Pan C, et al. Abnormal spontaneous brain activity in type 2 diabetic retinopathy revealed by amplitude of low-frequency fluctuations: a resting-state fMRI study. Clinical Radiology. 2017; 72: e1-e7.

[52] Inyushin M, Zayas-Santiago A, Rojas L, Kucheryavykh Y, Kucheryavykh L. Platelet-generated amyloid beta peptides in Alzheimer's disease and glaucoma. Histology and Histopathology. 2019; 34: 843-856. 